\title{
Analgesia for pain during subcutaneous injection: effectiveness of manual pressure application before injection
}

This article was published in the following Dove Press journal:

International Journal of General Medicine

24 August 2013

Number of times this article has been viewed

\section{Yutaka Nakashima' \\ Masanori Harada' \\ Masanobu Okayama ${ }^{2}$ \\ Eiji Kajii ${ }^{2}$}

'Department for Support Rural Medicine, Yamaguchi Grand Medical

Center, Yamaguchi, ${ }^{2}$ Division of Community and Family Medicine, Center for Community Medicine, Jichi Medical University, Tochigi, Japan
Correspondence: Yutaka Nakashima Yamaguchi Grand Medical Center, 77 Ohsaki, Hofu, Yamaguchi 747-85II, Japan

Tel +8I 8352244 I I

Fax +81835382210

Email nakashima.yutaka@ymghp.jp
Background: It is necessary to establish an effective subcutaneous injection procedure for adult and elderly individuals because many drugs such as hormones and interferon are generally delivered by subcutaneous injection. We tested whether pain during subcutaneous injection can be decreased by prior application of localized manual pressure at the injection site.

Methods: In this semirandomized, open-label study evaluating the manual pressure method for transient analgesia, physicians applied pressure with their thumbs for 10 seconds to create a nonpainful skin depression at the injection site immediately before subcutaneous injection of the influenza vaccine to patients. Control patients received the vaccine by the same route, but without prior application of focal pressure. In addition to pain, we evaluated patient age, gender, height, weight, body mass index, body temperature, and fat thickness at the brachial triceps muscle. Pain intensity was estimated using a $100 \mathrm{~mm}$ visual analog scale (VAS) and the face scale (FS). Categorical variables were compared using Chi-square tests and continuous variables were compared using unpaired $t$-tests between the intervention group and control group. Multivariate analysis was performed using the VAS or FS score as the dependent variable and weight, age, height, fat thickness at the brachial triceps muscle, and body temperature as independent variables.

Results: There were no significant differences in demographic variables, VAS scores $(22.5 \pm 23.0$ versus $21.2 \pm 23.6, P=0.4)$, or FS scores $(2.5 \pm 2.1$ versus $2.4 \pm 2.1, P=0.4)$ between the intervention and control groups. There was a significant negative correlation between age and subjective pain intensity (VAS, $r=-0.32$; FS, $r=-0.28$ ).

Conclusion: The manual pressure method was not effective in decreasing pain during subcutaneous injection. Alternative methods of focal transient analgesia should be developed to improve vaccination rates and relieve anxiety associated with subcutaneous injection.

Keywords: pain, subcutaneous injection, pressure, visual analog scale, analgesia

\section{Introduction}

Many treatment regimens require occasional or routine subcutaneous injections. Vaccination programs for immune activation are vital for preventing the spread of infection and decreasing the severity of infection in individual patients. ${ }^{1}$ Vaccines can be administered orally or by inhalation; however, subcutaneous, intradermal, or intramuscular injections are still the most common routes of administration. ${ }^{2}$

Family physicians regard fear of injections as an impediment to immunization compliance in adults. Patient vaccination compliance may be decreased by the fear of pain during injection. Although there are many studies on pain during vaccination in children, ${ }^{3-6}$ there are few studies focusing on the adult population, ${ }^{7,8}$ possibly because pain and fear of injection are presumed to be less intense in adults. ${ }^{3}$ These studies 
show that pain during vaccination can be decreased by the use of analgesic drugs. ${ }^{4,5}$ In addition, different types of physical interventions ${ }^{6}$ have been developed to decrease pain during injection. However, time constraints, expense, and the possibility of side effects have limited the application of these interventions. Therefore, they are unfamiliar in general practice.

In the United States and Europe, influenza vaccination is usually administered by intradermal injection. ${ }^{9}$ However, in Japan, influenza vaccination guidelines recommend subcutaneous injection. ${ }^{10}$ In adults, application of local manual pressure at the injection site decreases pain during subsequent intramuscular injection. ${ }^{8}$ This manual pressure method is advantageous because it requires no additional medication, has few (if any) side effects, requires no additional equipment, and does not appreciably prolong treatment time. Therefore, this method can be an effective and practical means of decreasing pain during subcutaneous injection.

In Japan, although the manual pressure method is recommended for relieving pain during injection of the influenza vaccine, no study has assessed the efficacy of this method for the relief of pain during subcutaneous injection. To evaluate the pain-relieving effects of this procedure, we compared visual analog scale (VAS) scores of adult patients who received subcutaneous injection immediately after 10 seconds of local pressure application at the injection site with those of controls who received no such treatment before injection.

\section{Materials and methods Study design}

This semirandomized, open-label study was conducted from November 11, 2011 to December 31, 2012 in rural clinics and general hospitals. The study protocol was approved by the research ethics committee of Yamaguchi Grand Medical Center and conformed to the principles of the Declaration of Helsinki. Patients were assured that their participation was voluntary, and all participants provided written informed consent.

\section{Participants}

Participants were adults ( $>20$ years of age) from the general population who were scheduled to receive the influenza vaccination by subcutaneous injection. Patients were excluded if they did not provide written consent, were aged $<20$ years, or could not complete the pain scales or other test forms. Patients who did not complete either were excluded from the analysis.

\section{Interventions}

The patients were semirandomly allocated to either the control (odd) or intervention (even) groups using case numbers. From a previous study, we estimated that the E/S (standard effect size/standard deviation) was $0.4{ }^{8}$ We estimated that the E/S for our study was 0.25 in consideration of the clinical effect and difference between subcutaneous injection and intramuscular injection. Values for $\alpha$ and $\beta$ were set at 0.05 and 0.20 , respectively. We estimated that 253 cases would be the size of each group. We set the target number for each group as 350 cases and a total of 700 cases in consideration of the exclusion criteria. Participants were not aware of their group allocation in advance, and the physician was not present during completion of the test measures for pain. The experimental intervention used in this trial was the manual pressure method, which was applied immediately before injection. Using this method, the physician applied pressure using the thumb to depress the injection site (posterior surface of the upper arm) for 10 seconds immediately before immunization. The pressure applied was sufficient to cause a skin depression but no pain. All other techniques were as per the 2003 influenza vaccination guidelines for $\operatorname{Japan}^{10}$ and included the unified even talking technique. The injection site was approximately one third down from the shoulder on the arm extensor of the patient's choice. The injection needle used for all participants measured $26 \mathrm{G} \times 0.45,1 / 2 \times 13 \mathrm{~mm}$. The vaccine used was acquired and stored by each clinic and hospital. The syringe was filled directly from the vial before vaccination.

\section{Measurements}

In addition to pain, we evaluated age, gender, height, weight, body mass index, body temperature, and fat thickness at the

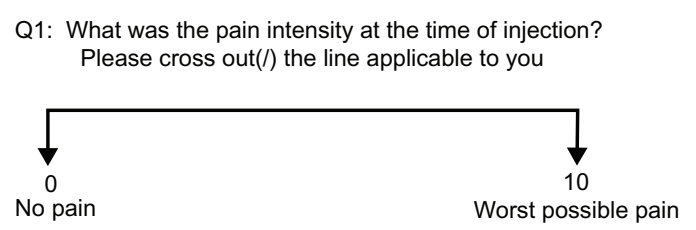

Q2: How was the pain at the time of injection? Please encircle (o) the picture applicable to you

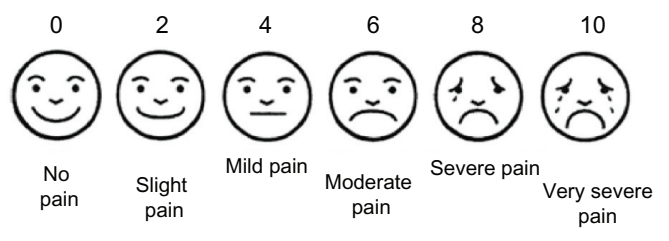

Figure I Pain questionnaire. 


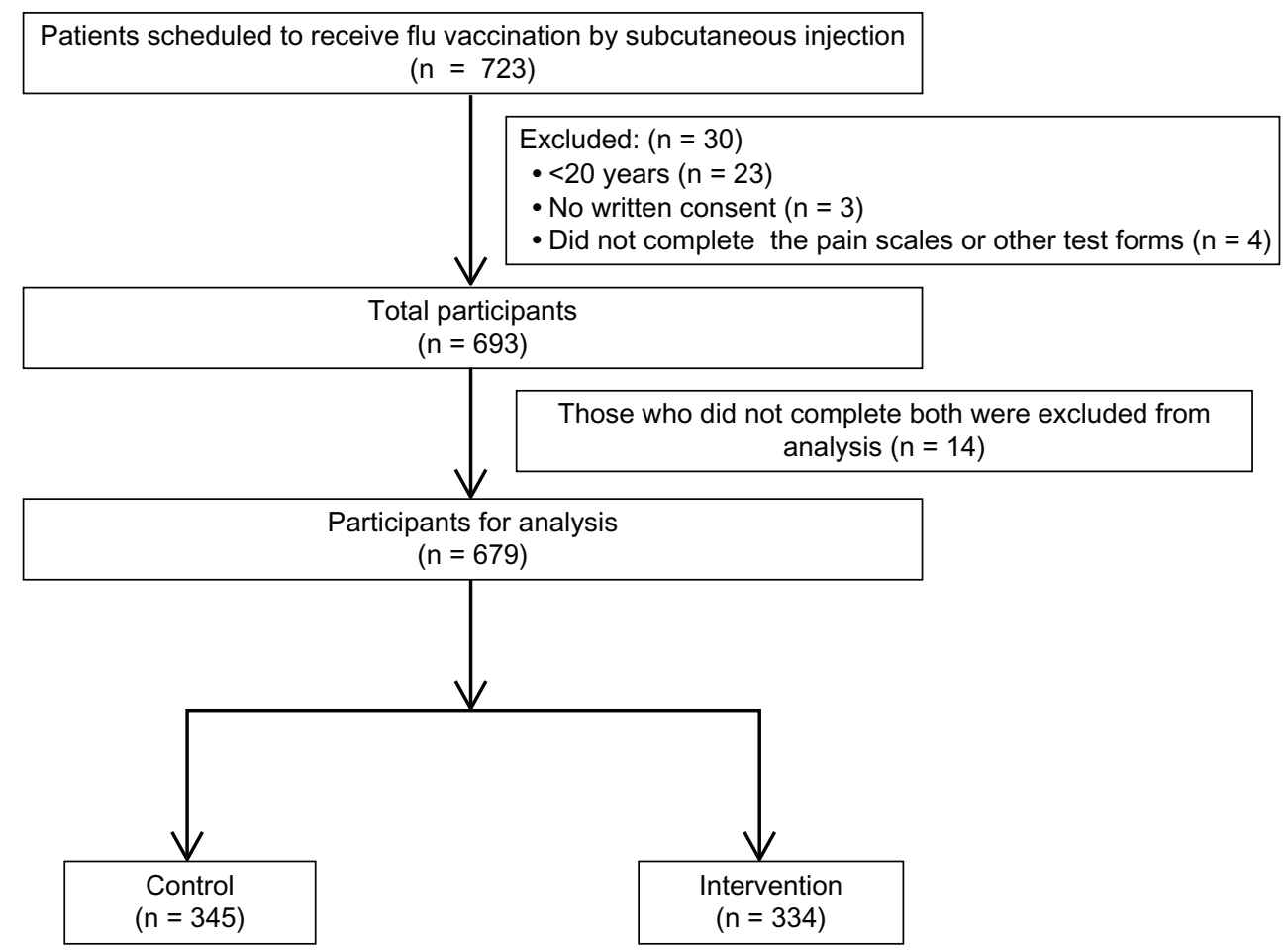

Figure 2 Selection of patients and randomization.

brachial triceps muscle. To assess pain, patients completed a $100 \mathrm{~mm}$ VAS and a face scale (FS, Figure 1).

\section{Statistical analysis}

We used the Statistical Package for the Social Sciences II (SPSS Japan Inc, Tokyo, Japan) for the statistical analysis. A $P$-value of $<0.05$ (two-tailed) was considered to be statistically significant. A correlation coefficient $(r)$ of $>0.2$ was considered significant. We calculated mean \pm standard deviations for categorical variables and proportions for continuous variables. Categorical variables were compared using Chi-square tests and continuous variables were compared using unpaired $t$-tests between the intervention and control groups. VAS and FS scores were treated as continuous variables. Multivariate analysis was performed using the VAS score or FS score as the dependent variable and weight, age, height, fat thickness at the brachial triceps muscle, and body temperature as independent variables.

\section{Results}

A total of 693 adults participated, of whom 679 were included in our analyses (345 in the intervention group and 334 in the control group, Figure 2). There were no significant differences in demographic or clinical variables between the two groups (Table 1). As a result, we analyzed 679 cases.
Therefore, the number of cases used was sufficient to consider the effectiveness of this method.

Although there were differences in the VAS $(22.5 \pm 23.0$ versus $21.2 \pm 23.6, P=0.4)$ and $\mathrm{FS}(2.5 \pm 2.1$ versus $2.4 \pm 2.1$, $P=0.4$ ) scores between the intervention and control groups, the differences were not significant (Table 2).

Multivariate analysis showed that there was a significant negative association between each pain score and age; younger participants reported more intense pain (VAS, $r=-0.32$;

Table I Baseline characteristics of patients in control and intervention groups

\begin{tabular}{|c|c|c|c|}
\hline & $\begin{array}{l}\text { Control } \\
(n=345)\end{array}$ & $\begin{array}{l}\text { Intervention } \\
(\mathrm{n}=334)\end{array}$ & $P$-value* \\
\hline Age, years, mean $\pm S D$ & $61.6 \pm 19.8$ & $61.4 \pm 18.7$ & 0.7 \\
\hline Gender, n (\%) & & & 0.4 \\
\hline Men & $105(30)$ & $91(27)$ & \\
\hline Women & $240(70)$ & $243(73)$ & \\
\hline Weight, kg, mean \pm SD & $54.8 \pm 10.3$ & $54.7 \pm 11.1$ & 0.6 \\
\hline Height, cm, mean \pm SD & $155 \pm 10.3$ & $154 \pm 10.6$ & 0.4 \\
\hline $\begin{array}{l}\text { Body mass index, } \mathrm{kg} / \mathrm{m}^{2} \\
\text { mean } \pm \mathrm{SD}\end{array}$ & $22.5 \pm 4.6$ & $22.8 \pm 4.3$ & 0.6 \\
\hline $\begin{array}{l}\text { Body temperature, }{ }^{\circ} \mathrm{C} \text {, } \\
\text { mean } \pm \mathrm{SD}\end{array}$ & $36.1 \pm 0.8$ & $36.1 \pm 0.5$ & 0.4 \\
\hline $\begin{array}{l}\text { Fat thickness at the } \\
\text { brachial triceps muscle, } \\
\mathrm{mm} \text {, mean } \pm S D\end{array}$ & $11.3 \pm 7.6$ & $11.8 \pm 7.8$ & 0.4 \\
\hline
\end{tabular}

Note: *Unpaired $t$-test or Chi-square test. Abbreviation: SD, standard deviation. 
Table 2 Visual analog scale and face scale scores for control and intervention (manual pressure method) groups

\begin{tabular}{lccl}
\hline & Control & Intervention & P-value* \\
\hline Visual analog scale score & $21.2 \pm 23.6$ & $22.5 \pm 23.0$ & 0.4 \\
Face scale score & $2.4 \pm 2.1$ & $2.5 \pm 2.1$ & 0.4 \\
\hline
\end{tabular}

Notes: Data are expressed as the mean \pm standard deviation. *Unpaired $t$-test.

FS, $r=-0.28)$. There were no other significant correlations between demographic or clinical variables and pain scores.

\section{Discussion}

The manual pressure method was not effective for decreasing pain during subcutaneous injection of the influenza vaccine. However, we clarified that the degree of pain associated with this influenza vaccine was judged to be mild (approximately $2 / 10$ ) as per the VAS score. Therefore, it remains to be determined if this method is more efficacious for more painful injections. There were significant differences in the level of pain experienced by participants of different ages, with younger participants reporting more intense pain.

Analgesia from manual pressure presumably involves interference of nociceptive transmissions by other somatosensory modalities, as explained by the gate control theory. ${ }^{11} \mathrm{Of}$ course, this study neither confirms nor refutes the gate control theory. Rather, we suggest that pain during subcutaneous injection is equally influenced by psychologic factors as it is by nociceptive stimulation, and older people and patients with diabetes have a higher pain threshold, probably because their receptors are worn out.

It was reported that pain during intramuscular injection was significantly more intense in women than in men, although there was no significant age dependence in this finding. ${ }^{7}$ In that study, the average age was lower and the age range narrower than in our study. In contrast, we found no gender differences in the level of pain experienced in our study, at least in the age group $<65$ years.

Our study has two limitations. First, we only assessed pain experienced during injection and not the subsequent pain that many experience at the injection site. Second, there was no way of masking the treatment, and pain ratings were subjective, leading to the possibility of a placebo effect.

International Journal of General Medicine

\section{Publish your work in this journal}

The International Journal of General Medicine is an international, peer-reviewed open-access journal that focuses on general and internal medicine, pathogenesis, epidemiology, diagnosis, monitoring and treatment protocols. The journal is characterized by the rapid reporting of reviews, original research and clinical studies across all disease areas.

\section{Conclusion}

The manual pressure method used in this study was not effective for decreasing mild pain during subcutaneous injection of the influenza vaccine. Pain during subcutaneous injection of the influenza vaccine was low (VAS $<3$ ), to the point that analgesia was not required. If necessary, we can consider the use of topical anesthetics as well as psychologic support for patients with extreme fear of needles.

\section{Acknowledgment}

We thank Kei Yokota, Kazuya Tanabe, Mitsue Nakazumi, Sachiko Kishinami, Fukiko Ishizi, and Satoko Tanaka for their assistance with data collection.

\section{Disclosure}

The authors report no conflicts of interest in this work.

\section{References}

1. Harpaz R, Ortega-Sanchez IR, Seward JF. Advisory Committee on Immunization Practices (ACIP) Centers for Disease Control and Prevention (CDC). MMWR Recomm Rep. 2008;57:1-30.

2. Krouger AT, Sumaya CV, Pickering LK, et al. Advisory Committee on Immunization Practices (ACIP) Centers for Disease Control and Prevention (CDC). MMWR Recomm Rep. 2011;60:1-60.

3. von Baeyer CL, Marche TA, Rocha EM, et al. Children's memory for pain: overview and implications for practice. J Pain. 2004;5:241-249.

4. O'Brien L, Taddio A, Ipp M, et al. Topical $4 \%$ amethocaine gel reduces the pain of subcutaneous measles-mumps-rubella vaccination. Pediatrics. 2004;114:e720-e724.

5. Halperin SA, McGrath P, Smith B, et al. Lidocaine-prilocaine patch decreases the pain associated with the subcutaneous administration of measles-mumps-rubella vaccine but does not adversely affect the antibody response. J Pediatr. 2000;136:789-794.

6. Berberich FR, Landman Z. Reducing immunization discomfort in 4- to 6-year-old children: a randomized clinical trial. Pediatrics. 2009;124: e203-e209.

7. Nahm FS, Lee PB, Park SY, et al. Pain from intramuscular vaccine injection in adults. Rev Med Chile. 2012;140:192-197.

8. Chung JW, Ng WM, Wong TK. An experimental study on the use of manual pressure to reduce pain in intramuscular injections. J Clin Nurs. 2002;11:457-461.

9. Belshe RB, Newman FK, Cannon J, et al. Serum antibody responses after intradermal vaccination against influenza. NEngl J Med. 2004;351: 2286-2294

10. Minister of Health, Labor and Welfare.org. Japan: the committee developing the guideline for flu vaccination. The guideline for flu vaccination. Available from: http://www-bm.mhlw.go.jp/topics/bcg/tp1107-1e.html. Accessed June 17, 2013.

11. Melzack R, Wall PD. Pain mechanism: a new theory. Science. 1965;150: 170-179.

\section{Dovepress}

A key focus is the elucidation of disease processes and management protocols resulting in improved outcomes for the patient.The manuscript management system is completely online and includes a very quick and fair peer-review system. Visit http://www.dovepress.com/ testimonials.php to read real quotes from published authors. 